\title{
The equivalence theorem and the production of gravitinos after inflation
}

\author{
Antonio L. Maroto* \\ CERN Theory Division, CH-1211 Geneva 23, Switzerland \\ J. R. Peláez \\ Departamento de Física Teórica, Universidad Complutense, 28040 Madrid, Spain
}

(Received 13 December 1999; published 26 June 2000)

\begin{abstract}
We study the high-energy equivalence between helicity $\pm 1 / 2$ gravitinos and Goldstinos in order to calculate the production of gravitinos in time-dependent scalar and gravitational backgrounds. We derive this equivalence for equations of motion, paying attention to some subtleties, mainly due to external sources, that are not present in the standard proofs. We also propose the Landau gauge as a simplifying alternative to the usual gauge choices, both for practical calculations and in the equivalence theorem proof.

PACS number(s): 98.80.Cq, 04.62.+v, 04.65.+e, 12.60.Jv
\end{abstract}

\section{INTRODUCTION}

In supergravity theories $[1,2]$ the graviton superpartner is a spin $3 / 2$ particle called the gravitino. This particle couples only with gravitational strength to the rest of matter fields, and accordingly its lifetime can be very long, with a decay rate of $\Gamma_{3 / 2} \simeq m_{3 / 2}^{3} / M_{P}^{2}$. In particular, gravitinos lighter than $m_{3 / 2}<100 \mathrm{MeV}$ will live longer than the age of the Universe. This fact can have important consequences in cosmology and imposes stringent constraints on supergravity models. Owing to their weak couplings, gravitinos freeze out very early when they are still relativistic; therefore their primordial abundance can be estimated as $n_{3 / 2} / s \simeq 10^{-3}$ [3]. Considering only the case of unstable gravitinos, such a primordial abundance would give rise to an enormous amount of entropy, in conflict with the standard cosmology. In particular, gravitinos decaying during the nucleosynthesis can destroy the nuclei created in that era. A possible way out of this gravitino problem is the existence of a period of inflation that dilutes any primordial density [4]. Unfortunately the problem can be re-created if, after inflation, gravitinos are produced by some mechanism. In fact, this could be the case if during the period of inflaton oscillations, at the end of inflation, the reheating temperature was sufficiently high. A successful nucleosynthesis era then requires (we give some conservative bounds [5]) $n_{3 / 2} / s<10^{-15}$ for a gravitino mass $m_{3 / 2} \simeq 100 \mathrm{GeV}, n_{3 / 2} / s<10^{-14}$ for $m_{3 / 2} \simeq 1 \mathrm{TeV}$ and $n_{3 / 2} / s$ $<10^{-13}$ for $m_{3 / 2} \simeq 10 \mathrm{TeV}$. The production of gravitinos during reheating is due to processes involving other particles produced from the inflaton decay, and depends on the reheating temperature $T_{R}$ as [6]: $\quad n_{3 / 2} / s \simeq 10^{-14} T_{R} /$ $\left(10^{9} \mathrm{GeV}\right)$. For a typical mass $m_{3 / 2} \simeq 1 \mathrm{TeV}$, this implies $T_{R}<10^{9} \mathrm{GeV}$. Another constraint appears in supergravity models where the gravitino mass is determined by the scale of supersymmetry breaking. In order to solve the hierarchy problem, it is then suggested that $m_{3 / 2}<1 \mathrm{TeV}$ [2].

However, as a consequence of some recent works [7] it

\footnotetext{
*Email address: Antonio.Lopez.Maroto@cern.ch

${ }^{\dagger}$ Email address:pelaez@eucmax.sim.ucm.es
}

was realized that, during the first inflaton oscillations, reheating cannot be studied by the standard perturbative techniques. This preheating period can give rise to an explosive production of bosons due to the phenomenon of parametric resonance. In this period, the energy of the coherent oscillations of the inflaton field is very efficiently converted into particles. In the case of fermions, the limit imposed by the Pauli exclusion principle avoids the explosive production, although the results still deviate from the perturbative expectations $[8,9]$. This fact is particularly relevant when gravitinos are directly coupled to the inflaton, since during preheating they could be produced in excess, thus imposing new constraints on the particular supergravity inflationary model.

In previous works $[10,11]$ it was shown that the production of helicity $\pm 3 / 2$ gravitinos can take place during preheating and that the results deviate from the perturbative expectations by several orders of magnitude (see also [12]). In the case of helicity $\pm 1 / 2$ gravitinos, the production is in general more abundant, depending on the specific supergravity model [13]. Some other works dealing with this topic can be found in [14].

In the present work we are interested in the production of helicity $\pm 1 / 2$ gravitinos during preheating. The relative difficulty of the calculations in the unitary gauge, used in the above references, suggests that we should explore alternative methods. In particular we will exploit the relation between helicity $\pm 1 / 2$ gravitinos and Goldstinos (first pointed out in Ref. [28]) given by the equivalence theorem (ET) [15]. This possibility was suggested in the first reference of [13] and in the last one of [13] it was shown how the ET could be used to study the helicity $\pm 1 / 2$ gravitino equation in the $|\phi|$ $\ll M_{P}$ limit.

The ET was first introduced, in the framework of nonAbelian gauge theories $[16,17]$, as a way to calculate processes involving longitudinal gauge bosons, but using only Goldstone bosons, which are scalars and therefore much easier to handle. The first formal proof in terms of $S$-matrix elements was given in [18], and that is basically the derivation followed within the supergravity scenario. In the last few years, and still within the framework of the non-Abelian gauge theory, several works have completed the proof of the theorem [19], including renormalization effects, but also raising some questions about its Lorentz non-invariance am- 
biguity and applicability [20]. In this paper we also discuss briefly how these new considerations may affect the gravitino-Goldstino ET applied to the production of gravitinos during preheating.

Intuitively, the ET tells us that, since the Goldstinos disappear from the spectrum through the super-Higgs mechanism, giving rise to physical helicity $\pm 1 / 2$ gravitinos, it is possible to use Goldstinos in the calculation of observables instead of the complicated $\pm 1 / 2$ gravitinos. Of course, this identification can only be carried out at energies high enough to neglect the masses.

Rigorously, this theorem has only been proved for $S$-matrix elements containing initial or final helicity $\pm 1 / 2$ gravitinos and in the absence of external backgrounds. This would provide a good approximation for gravitino production during the reheating period, but only at the perturbative level, where the rate of production is given by the decay of inflaton quanta $[7,15]$.

However, preheating is a non-perturbative (and out of equilibrium) process and it is not obvious that the same proof still holds in the presence of external sources, such as the inflaton field or the space-time curvature. In particular, the presence of a source that creates particles makes different the initial and final vacua in the Green functions. In addition, these sources are present in the gauge-fixing condition, which is the starting relation in the ET derivations. Of course, we still expect that the intuitive relation suggested by the ET should hold, but since it is not the same to establish an equality at the level of matrix elements as at the operator (fields, indeed) level, we present in this paper a derivation more suited for the formalism in terms of equations of motion. In this way we can also identify the physical conditions on the sources that we need for this theorem to hold.

Finally, we propose the Landau gauge as the best choice to perform the calculations, although, probably, it is not the most intuitive. In this gauge, not only the proof of the theorem, but also the final equations that govern gravitino production are considerably simpler.

All the previous considerations basically concern the gravitino production process. But we also have to take into account the fact that we are producing very many gravitinos (out of equilibrium) which have a distribution in energies. Some of them will satisfy the physical conditions to apply the ET, whereas some others will not. Hence, we also present an additional condition on the number of those gravitinos not satisfying the applicability conditions, in order to obtain reliable calculations with the ET.

\section{SUPERGRAVITY LAGRANGIAN}

Let us consider $N=1$ minimal supergravity $[1,2,21]$ coupled to a single chiral superfield $\Phi$, which describes a complex scalar field $\phi$ and a Majorana spinor $\eta$ satisfying $\eta=C \bar{\eta}^{T}=\eta^{C}$, with the charge-conjugation matrix given by $C=i \gamma^{2} \gamma^{0}$. In principle, the derivation could be extended to more than one chiral multiplet in a similar way. The scalar component will play the role of the inflaton field and it will therefore be considered as an external background. The corresponding Lagrangian is defined by the superpotential
$W(\Phi)$ and the Kähler potential $G\left(\Phi, \Phi^{\dagger}\right)=\Phi^{\dagger} \Phi+\log |W|^{2}$. We will define: $G_{, \phi}=\partial G / \partial \phi, G_{, \phi^{*}}=\partial G / \partial \phi^{*}, G_{, \phi \phi^{*}}$ $=\partial^{2} G / \partial \phi \partial \phi^{*}$, etc. In this case we will have $G_{, \phi \phi^{*}}=1$. The bosonic part of the Lagrangian is given by

$$
g^{-1 / 2} \mathcal{L}_{B}=-\frac{1}{2} R+g^{\mu \nu} \partial_{\mu} \phi \partial_{\nu} \phi^{*}+e^{G}\left(3-\left|G_{, \phi}\right|^{2}\right),
$$

where we are working in units $M=M_{P} / \sqrt{8 \pi}=1$. In the fermionic part of the Lagrangian, we are only interested in those terms quadratic in the fermionic fields (gravitinos and Goldstinos), since we are going to work with the linearized equations of motion. For the sake of simplicity we will assume that the scalar field $\phi$ is real. With this assumption those terms are:

$$
\begin{aligned}
g^{-1 / 2} \mathcal{L}_{F}= & -\frac{1}{2} \epsilon^{\mu \nu \rho \sigma} \bar{\psi}_{\mu} \gamma_{5} \gamma_{\nu} D_{\rho} \psi_{\sigma}+\frac{i}{2} \bar{\eta} I D \eta \\
& +e^{G / 2}\left(\frac{i}{2} \bar{\psi}^{\mu} \sigma_{\mu \nu} \psi^{\nu}+\frac{1}{2}\left(-G_{, \phi \phi}-G_{, \phi}^{2}\right) \bar{\eta} \eta\right. \\
& \left.+\frac{i}{\sqrt{2}} G_{, \phi} \bar{\psi}_{\mu} \gamma^{\mu} \eta\right)+\frac{1}{\sqrt{2}} \bar{\psi}_{\mu}(\not \partial \phi) \gamma^{\mu} \eta,
\end{aligned}
$$

with $\sigma_{\mu \nu}=i / 2\left[\gamma_{\mu}, \gamma_{\nu}\right]$. Since we are concerned with the production of gravitinos after inflation, we assume that our scalar field depends only on time and that the space-time metric is of the Friedmann-Robertson-Walker (FRW) form. In particular, it will be very useful to work in conformal time, for which the FRW metric with flat spatial sections reads

$$
d s^{2}=a^{2}(t)\left(d t^{2}-d \vec{x}^{2}\right),
$$

where $a(t)$ is the Universe scale factor and the nonvanishing gravitational field is assumed to be created by the scalar field.

In contrast with the ET usual proof, there are two mixing terms between gravitinos and Goldstinos in Eq. (2). When the scalar field has settled down at the potential minimum, $\phi=\phi_{0}$, the last term does not contribute, and this is why it is absent from the discussions of the spontaneous breaking of supersymmetry. However, since we are interested in a timedependent $\phi$, such a term cannot be ignored any longer. In flat space-time, with $\phi=\phi_{0}$, and when supersymmetry is not broken, i.e., $G_{, \phi_{0}}=0$, the mixing terms are absent and the equations of motion describe the gravitino evolution with only two helicity $\pm 3 / 2$ states. However, when supersymmetry is broken spontaneously, the gravitino acquires two more degrees of freedom with helicity $\pm 1 / 2$, because of the interaction with the Goldstinos, giving rise to much more complicated evolution equations.

In the unitary gauge all the Goldstino dependent terms are absorbed in a redefinition of the gravitino field. This gauge shows explicitly the super-Higgs mechanism in which the Goldstino becomes the helicity $\pm 1 / 2$ components of the gravitino field. There are no mixing terms but, still, we have to deal with $\pm 1 / 2$ helicity states of a Rarita-Schwinger field, 
which can be rather involved. Nevertheless, the production of helicity $\pm 1 / 2$ gravitinos in preheating has been calculated in the unitary gauge in [13].

Note that, although it is not necessary, we are making the inflaton responsible for supersymmetry breaking. Then, the inflatino also plays the role of the Goldstino. This assumption simplifies the discussion since otherwise, and although supersymmetry would be broken during and after inflation, it would be restored at the minimum of the potential and the super-Higgs mechanism would not take place. Accordingly, the gravitino would not have a $\pm 1 / 2$ component. We will also assume that at the minimum the cosmological constant is zero, then $G_{, \phi_{0}}^{2}=3 G_{, \phi_{0} \phi_{0}^{*}}$, and we have $G_{, \phi_{0}}=\sqrt{3}$.

The equations of motion for gravitinos and Goldstinos derived from Eq. (2) are

$$
\begin{gathered}
\epsilon^{\mu \nu \rho \sigma} \gamma_{5} \gamma_{\nu} D_{\rho} \psi_{\sigma}+\frac{1}{2} e^{G / 2}\left[\gamma^{\mu}, \gamma^{\nu}\right] \psi_{\nu}-\frac{i}{\sqrt{2}} G_{, \phi} e^{G / 2} \gamma^{\mu} \eta \\
-\frac{1}{\sqrt{2}}(\not \phi) \gamma^{\mu} \eta=0
\end{gathered}
$$

and

$$
\begin{aligned}
i \not D & \eta+e^{G / 2}\left(-G_{, \phi \phi}-G_{, \phi}^{2}\right) \eta-\frac{i}{\sqrt{2}} e^{G / 2} G_{, \phi} \gamma^{\mu} \psi_{\mu} \\
& +\frac{1}{\sqrt{2}} \gamma^{\mu}(\not \phi) \psi_{\mu}=0 .
\end{aligned}
$$

If we consider only helicity $\pm 3 / 2$ gravitinos, then it can be shown that the equations of motion reduce to a very simple form [10]:

$$
\left(i \not D-e^{G / 2}\right) \psi_{\mu}^{ \pm 3 / 2}=0
$$

However, the helicity $\pm 1 / 2$ equation is much more involved and contains terms coupled to Goldstinos. Nevertheless, if we are only interested in the helicity $\pm 1 / 2$ gravitinos highenergy behavior, $E \gg m_{3 / 2}$, then we can simplify the calculations with the ET. This limit is sensible in most supergravity inflation models with one chiral supermultiplet, since the typical energy of the particles created during preheating is of the order of the inflaton mass, $m_{\phi}$, which is usually several orders of magnitude larger than $m_{3 / 2}$. For instance, in the model discussed in $[22,23], m_{\phi} \simeq 10^{10} \mathrm{GeV}$, whereas $m_{3 / 2}$ $<1 \mathrm{TeV}$. Note also that all these scales are well below $M_{P}$, where supergravity breaks down as an effective theory.

As it was commented before, the ET has been rigorously derived for S-matrix elements. However, to calculate the non-perturbative production of gravitinos during preheating, we use a formalism in terms of equations of motion and fields. In order to show how the gravitino-Goldstino highenergy equivalence can be used in this context, we will follow these steps: (i) Introduce a gauge-fixing term corresponding to a certain generalization of the $R_{\xi}$ gauges, which allows us to cancel the mixing gravitino-Goldstino terms in the equations of motion.

(ii) Assume that in the asymptotic regions $t \rightarrow \pm \infty$, the external sources are static, i.e., $\phi \rightarrow \phi_{0}$ and $g_{\mu \nu} \rightarrow \eta_{\mu \nu}$, and then use the equations of motion in those regions to show that $\partial^{\mu} \psi_{\mu} \propto m_{3 / 2} \eta$.

(iii) Use the high-energy limit of the $\pm 1 / 2$ helicity projectors, $\quad P_{ \pm 1 / 2}^{\mu}=p^{\mu} / m_{3 / 2}+\mathcal{O}\left(m_{3 / 2} / E\right)$, to relate $\psi_{ \pm 1 / 2}$ $=P_{ \pm 1 / 2}^{\mu} \psi_{\mu} \propto \eta$, when $E \gg m_{3 / 2}$ in the asymptotic regions.

(iv) Choose the Landau gauge, $\xi \rightarrow \infty$, as an additional simplification for the calculations of Goldstino production.

\section{GAUGE FIXING}

Goldstinos do not belong to the physical spectrum, and in the unitary gauge we can even get rid of them in the equations of motion. In contrast, the production of helicity $\pm 1 / 2$ gravitinos during reheating is gauge-invariant, and is only related to the Goldstino production in certain gauges, called $R_{\xi}$ gauges, in which both fields appear simultaneously in the Lagrangian. Let us then consider the following gauge-fixing condition, which is a generalization of the $R_{\xi}$ gauge used in $[15,24]$ :

$$
\gamma^{\mu} \psi_{\mu}-\frac{1}{\sqrt{2} \xi \not D} e^{G / 2} G_{, \phi} \eta+\frac{i}{G_{, \phi}} e^{-G / 2} \gamma^{\mu}(\not \phi) \psi_{\mu}=0 .
$$

When $\phi$ is constant we recover the gauge-fixing term in [15] and the limit $\xi \rightarrow 0$ corresponds to the unitary gauge. Note that in our case, due to the external sources, all the coefficients in the gauge-fixing function are no longer constants. The above equation provides us with a relation between gravitinos and Goldstinos, but we want to extract only those with helicity $\pm 1 / 2$, for which we will need a relation between $\partial^{\mu} \psi_{\mu}$ and $\eta$. In the following we will use the equations of motion to obtain a relation of the desired form.

If we assume that in the asymptotic regions $t \rightarrow \pm \infty$ the space-time is flat and the scalar field settles down at the potential minimum $\phi_{0}$, then, in those regions, the above condition reduces to

$$
a_{\text {in, out }}^{-1} \theta \gamma^{\mu} \psi_{\mu}=\sqrt{\frac{3}{2}} \frac{m_{3 / 2}}{\xi} \eta,
$$

where $m_{3 / 2}=e^{G_{0} / 2}$ and $a_{\text {in,out }}$ are the scale factor values in the asymptotic past and future. In order to simplify the notation, we will absorb the scale factor into the mass: $m_{\text {in,out }} \equiv a_{\text {in, out }} m_{3 / 2}$; to avoid the proliferation of indices, we will denote $m_{\text {in,out }}$ simply by $m$. With this notation, the gauge-fixing condition reads

$$
b \gamma^{\mu} \psi_{\mu}=\frac{m}{\xi} \sqrt{\frac{3}{2}} \eta
$$

Let us recall that it is only in the static regions where the definition of particle and the separation between different helicities is unambiguous. However, in the strict sense, 
within the inflationary cosmology neither the initial $(t \rightarrow$ $-\infty)$ nor the final $(t \rightarrow \infty)$ regions can be considered static, since there is a period of inflation before preheating and today we know that the Universe is expanding. Nevertheless, for practical purposes, we can still consider the initial and final regions as static, since the particle production will mainly take place during the first inflaton oscillations. Accordingly, we will define our initial vacuum by imposing such initial conditions on our fields that they behave as plane waves before preheating. The final state has a similar behavior, since the rate of expansion decreases with time. Indeed, the vacuum at the end of preheating could be defined more rigorously as an adiabatic vacuum [25], which would not yield additional gravitino production from the Universe expansion.

Let us then consider first the equations of motion for gravitinos (4) and Goldstinos (5) in the initial and final regions with the notation that we have just introduced. Since the inflaton is in the minimum, $G_{, \phi_{0}^{*}} G_{, \phi_{0} \phi_{0}}=-G_{, \phi_{0}}$, and therefore

$$
\begin{gathered}
\epsilon^{\mu \nu \rho \sigma} \gamma_{5} \gamma_{\nu} \partial_{\rho} \psi_{\sigma}+\frac{1}{2} m\left[\gamma^{\mu}, \gamma^{\nu}\right] \psi_{\nu}-i \sqrt{\frac{2}{3}} m \gamma^{\mu} \eta=0 \\
i \not \eta \eta-2 m \eta-i \sqrt{\frac{3}{2}} m \gamma^{\mu} \psi_{\mu}=0 .
\end{gathered}
$$

If we now fix the gauge using Eq. (9) in the above equations, they can be rewritten as

$$
\begin{gathered}
\epsilon^{\mu \nu \rho \sigma} \gamma_{5} \gamma_{\nu} \partial_{\rho} \psi_{\sigma}+\frac{1}{2} m\left[\gamma^{\mu}, \gamma^{\nu}\right] \psi_{\nu}-i \xi \gamma^{\mu} \partial \gamma^{\nu} \psi_{\nu}=0 \\
i \not \partial \eta-2 m \eta-i \frac{3}{2} \frac{m^{2}}{\xi} \frac{1}{b} \eta=0
\end{gathered}
$$

In the following we will rewrite the equations of motion for Goldstinos and gravitinos in the asymptotic regions as well as the gauge-fixing condition in a more convenient form. Contracting the gravitino equation with $\partial_{\mu}$, we obtain [26]

$$
\frac{1}{2} m\left(\partial \gamma^{\nu} \psi_{\nu}-\gamma^{\nu} \partial \psi_{\nu}\right)-i \xi \partial \partial \gamma^{\nu} \psi_{\nu}=0
$$

whereas contracting with $\gamma_{\lambda} \gamma_{\mu}$, we find

$$
2 i\left(\partial_{\lambda} \gamma^{\sigma} \psi_{\sigma}-\not \partial \psi_{\lambda}\right)+m\left(\gamma_{\lambda} \gamma^{\nu} \psi_{\nu}+2 \psi_{\lambda}\right)-2 i \xi \gamma_{\lambda} \partial \gamma^{\nu} \psi_{\nu}=0
$$

which can be contracted again with $\gamma_{\lambda}$ to get

$$
i\left(\not \partial \gamma^{\sigma} \psi_{\sigma}-\gamma^{\lambda} \not \partial \psi_{\lambda}\right)+3 m \gamma^{\mu} \psi_{\mu}-4 i \xi \partial \gamma^{\nu} \psi_{\nu}=0 .
$$

Substituting Eq. (14) into Eq. (16), we obtain

$$
-i \xi \partial \partial \gamma^{\nu} \psi_{\nu}+\frac{3}{2} i m^{2} \gamma^{\nu} \psi_{\nu}+2 \xi m b \gamma^{\nu} \psi_{\nu}=0,
$$

which, finally, can be rewritten as

$$
\left(i \not b-m_{+}\right)\left(i \not \partial-m_{-}\right) \gamma^{\nu} \psi_{\nu}=0
$$

where we have defined $m_{ \pm}=m(1 \pm \sqrt{1-3 /(2 \xi)})$. Note that, in the perturbative sense, the poles in the propagator are exactly those obtained in [15]. In addition we can derive the very same equation for the Goldstino, just by multiplying Eq. (13) by $i b$ :

$$
\left(i \not \partial-m_{+}\right)\left(i \not \partial-m_{-}\right) \eta=0 .
$$

The implications of these equations are clearer if we recall that the physical fields, i.e., the $\pm 3 / 2$ and $\pm 1 / 2$ helicity modes in the asymptotic regions, are those satisfying both $\gamma^{\mu} \psi_{\mu}=0$ and $\partial^{\mu} \psi_{\mu}=0$. They have the correct physical mass $m$ since they still satisfy $(i \not b-m) \psi_{\mu}^{P h y}=0$. In contrast, the unphysical spin-1/2 modes present poles in the propagator at $m_{ \pm}$, exactly as happens with the Goldstinos. Therefore, by fixing the gauge we have only modified the poles of the unphysical modes. From Eq. (14) and by means of the gauge-fixing condition, we obtain

$$
\frac{1}{2} m\left(2 \not \gamma^{\nu} \psi_{\nu}-2 \partial^{\nu} \psi_{\nu}\right)-i \partial \sqrt{\frac{3}{2}} m \eta=0 .
$$

Since $\eta$ satisfies Eq. (19), we have two possible solutions: $\left(i \not b-m_{+}\right) \eta=0$ and $\left(i \not b-m_{-}\right) \eta=0$; together with the gauge-fixing condition (20), they yield

$$
\sqrt{\frac{3}{2}} \frac{m}{\xi} \eta-\partial^{\mu} \psi_{\mu}-\sqrt{\frac{3}{2}} m_{ \pm} \eta=0 .
$$

From this expression we get

$$
\partial^{\mu} \psi_{\mu}=\sqrt{\frac{3}{2}} \frac{m}{\xi}\left(1-\xi \frac{m_{ \pm}}{m}\right) \eta
$$

At first sight, this equation relates the unphysical gravitino $\partial^{\mu} \psi_{\mu}$ with the Goldstino; however, the key observation is that, as we will show, at high energy, $\partial^{\mu} \psi_{\mu}$ tends to the physical helicity $\pm 1 / 2$ gravitino. Note that, apparently, there are two relations, one for Goldstinos that correspond to the $m_{-}$solution and another for those with $m_{+}$.

\section{THE EQUIVALENCE THEOREM}

In the asymptotic initial and final regions, we expect that a general solution of the equations of motion for gravitinos and Goldstinos will be written as a linear superposition of on-shell positive and negative frequency plane waves [26]. In particular, let us consider a negative frequency mode solution in the initial region with momentum $p^{\mu}=\left(\omega_{i n}, 0,0, p\right)$ (there is no loss of generality in this choice), with $p_{\mu} p^{\mu}$ $=m_{i n}^{2}$ and $p=|\vec{p}|$, such that $p \gg m_{i n}$ : 


$$
\psi_{\mu}^{p}(x)=\frac{1}{a_{i n}^{3 / 2} \sqrt{2 \omega_{i n}}} e^{i p x} \widetilde{\psi}_{\mu}(\vec{p})+\mathcal{O}\left(\frac{m_{i n}}{p}\right),
$$

where $\widetilde{\psi}_{\mu}(\vec{p})$ is the corresponding Fourier component. For the unphysical Goldstino we have

$$
\eta^{p}(x)=\frac{1}{a_{i n}^{3 / 2} \sqrt{2 \omega_{i n}}} e^{i p x} \tilde{\eta}(\vec{p})+\mathcal{O}\left(\frac{m_{i n}}{p}\right) .
$$

Similar expressions can be written for the positive-frequency solutions and for solutions in the final region. Note that the on-shell conditions for gravitinos and Goldstinos are different because of the different positions of the poles. In particular, for physical gravitinos we have $p_{\mu} p^{\mu}=m_{i n}^{2}$ and for Goldstinos and unphysical gravitinos $\left(\gamma^{\mu} \psi_{\mu}\right.$ and $\left.\partial^{\mu} \psi_{\mu}\right)$ we have $p_{\mu} p^{\mu}=\left(m_{+}^{i n}\right)^{2}$ (we will not use $m_{-}$for reasons that will become clear below). We have thus included the $\mathcal{O}\left(m_{\text {in }} / p\right)$ term at the end of Eqs. (23) and (24). Strictly, it should not be there for physical gravitinos, but at this level we keep a compact notation between physical and unphysical gravitinos.

The spin-1 polarization vectors are given by $\epsilon_{\mu}(\vec{p}, m)$ $=a(t) \delta_{\mu}^{a} \epsilon_{a}(\vec{p}, m)$, where

$$
\begin{aligned}
\epsilon_{a}(\vec{p}, 1) & =\frac{1}{\sqrt{2}}(0,1, i, 0), \quad \epsilon_{a}(\vec{p}, 0)=\frac{1}{m_{\text {in }}}\left(p, 0,0, \omega_{\text {in }}\right), \\
\epsilon_{a}(\vec{p},-1) & =-\frac{1}{\sqrt{2}}(0,1,-i, 0) .
\end{aligned}
$$

If $u(\vec{p}, s)$ are spinors with definite helicity $s= \pm 1 / 2$, then $P_{ \pm} u(\vec{p}, \pm 1 / 2)=u(\vec{p}, \pm 1 / 2), \quad$ where $\quad P_{ \pm}=(1 / 2)(1$ $\left.\pm \gamma_{5} \gamma^{\mu} \epsilon_{\mu}(\vec{p}, 0)\right)$ are the helicity projectors. Accordingly, the helicity $\pm 3 / 2$ and $\pm 1 / 2$ projectors are nothing but

$$
\begin{aligned}
P_{\mu}^{ \pm 3 / 2}= & P_{ \pm} \epsilon_{\mu}(\vec{p}, \pm 1), \\
P_{\mu}^{ \pm 1 / 2}= & \sqrt{\frac{1}{3}} P_{\mp} \epsilon_{\mu}(\vec{p}, \pm 1) \\
& +\sqrt{\frac{2}{3}} P_{ \pm} \epsilon_{\mu}(\vec{p}, 0) .
\end{aligned}
$$

We see that, at high energy, the $\pm 1 / 2$ projector behaves as

$$
P_{\mu}^{ \pm 1 / 2}=\sqrt{\frac{2}{3}} P_{ \pm} \frac{p_{\mu}}{m_{\text {in }}}+\mathcal{O}\left(\frac{m_{\text {in }}}{p}\right)
$$

where we have neglected $\epsilon_{\mu}(\vec{p}, \pm 1)$ with respect to $\epsilon_{\mu}(\vec{p}, 0)$. Let us then define the helicity $\pm 1 / 2$ components of the gravitino field in the asymptotic initial regions and in momentum space as

$$
\widetilde{\psi}_{ \pm 1 / 2}(\vec{p}) \equiv P_{ \pm 1 / 2}^{\mu} \widetilde{\psi}_{\mu}(\vec{p})=\left[\sqrt{\frac{2}{3}} P_{ \pm} \frac{p^{\mu}}{m_{i n}}+\mathcal{O}\left(\frac{m_{i n}}{p}\right)\right] \widetilde{\psi}_{\mu}(\vec{p})
$$

At high energies, we see that the helicity $\pm 1 / 2$ gravitino tends to the unphysical $\partial^{\mu} \psi_{\mu}$ field and therefore we can use the gauge-fixing condition in Eq. (22) to obtain a relation between each Fourier mode of the Goldstino and the helicity $\pm 1 / 2$ gravitino. [As pointed out in [15], it is essential that both $\partial^{\mu} \psi_{\mu}$ and $\eta$ have the same poles, in order to rewrite the equality in Eq. (22) in terms of Fourier modes.]

For arbitrary values of the $\xi$ parameter it would be necessary to take into account both solutions, with $m_{+}$and with $m_{-}$. In this case, the solution of the Goldstino equation is

$$
\eta_{p}(x)=\eta_{p}^{+}(x)+\eta_{p}^{-}(x) .
$$

From Eq. (22), we see that each solution is related to the $1 / 2$ helicity gravitino with different proportionality constants, i.e., for negative frequency solution we will have in the initial region

$$
\begin{aligned}
\widetilde{\psi}_{ \pm 1 / 2}(\vec{p})= & \sum_{+,-}\left[-i \frac{1}{\xi}\left(1-\xi \frac{m_{+,-}^{i n}}{m_{\text {in }}}\right) P_{ \pm 1 / 2}\right. \\
& \left.+\mathcal{O}\left(\frac{m_{i n}}{p}\right)\right] \tilde{\eta}^{+,-}(\vec{p}) .
\end{aligned}
$$

In the $S$-matrix derivations of the ET, the proportionality constant between the helicity $\pm 1 / 2$ gravitinos and the Goldstinos disappears once the external lines of the Green functions have been removed, the momenta are on-shell, and the tensor indices are contracted with the corresponding polarization vectors [15]. However, this is not so straightforward in the "semiclassical" proofs based on the generating functional formalism, either within supergravity [15] or even in the non-Abelian context [16]. These "semiclassical" proofs are given for the clever choice $\xi=3 / 2$, where $m_{-}=m_{+}$and the proportionality constant is unique (in the non-Abelian case the choice is $\xi=1$ and the proportionality constant is unity).

However, for our purposes, it is much more appropriate to choose the Landau gauge. Indeed, in an arbitrary generalized $R_{\xi}$ gauge, Eq. (5) will be written

$$
i \text { ID } \eta-e^{G / 2}\left(G_{, \phi \phi}+G_{, \phi}^{2}\right) \eta-e^{G / 2} G_{, \phi} \frac{i}{2 \xi \not D} e^{G / 2} G_{, \phi} \eta=0 .
$$

The presence of the last term makes it very difficult to obtain solutions even numerically. However, we get a dramatic simplification by using the Landau gauge, $\xi \rightarrow \infty$, in which the last term, which is the most complicated, vanishes. Thus we have

$$
i \not D \eta-e^{G / 2}\left(G_{, \phi \phi}+G_{, \phi}^{2}\right) \eta=0 .
$$

Note that this last expression corresponds to the $m_{+}$case in Eq. (19). The $m_{-}=0$ solution is just an artifact due to the 
multiplication by $i b$ and we do not have to consider it in the previous formulas. Therefore, Eq. (28) now reads

$$
\widetilde{\psi}_{ \pm 1 / 2}(\vec{p})=\left[2 i P_{ \pm}+\mathcal{O}\left(\frac{m_{i n}}{p}\right)\right] \eta(\vec{p})
$$

This is the relation we were looking for. Note that this is an equality at the level of fields and not for $S$-matrix elements. This result shows that although the helicity $\pm 1 / 2$ gravitinos and the Goldstinos can evolve differently during the oscillations of the scalar field, they approach each other in the asymptotic regions (up to a constant). The fact that this result is valid only in the asymptotic regions, is sufficient for our purposes. Since Eq. (32) is just the standard equation of motion for a fermion field in curved space-time with a timedependent mass, it is straightforward to apply the standard techniques of particle production.

Once more we stress that such a result is only useful when the energy of the particles we are producing is much larger than their masses. In our case, the inflaton is in a frame where it is homogeneous, only depends on $t$, and oscillates with a typical frequency $m_{\phi}$. Thus, we expect the physical momentum of the gravitinos to be $\mathcal{O}\left(m_{\phi}\right)$, so that we can use Eq. (33) if $m_{3 / 2} \ll m_{\phi}$. Usually we will evaluate $a_{\text {out }}$ right after the preheating ends and then $m_{\text {in,out }} \ll m_{3 / 2}$. The fact that we are in an homogeneous background is technically relevant due to the remarks about the ET and Lorentz invariance done in [20] in the context of non-Abelian gauge theories. Indeed the ET is not Lorentz invariant, since not only is the helicity decomposition frame-dependent, but also terms like $\mathcal{O}(m / E), \mathcal{O}(m / p) \ldots$ do have very different values depending on the reference frame. The fact that the inflaton field is homogeneous ensures that all our gravitinos are produced from rather similar conditions and we can apply the ET to the vast majority of them.

\section{PARTICLE PRODUCTION}

Up to now our discussion has been purely classical. In order to interpret these solutions in terms of particle number, we have to quantize them [25,27], which has already been done in the unitary gauge [13]. We are interested in the Landau gauge, $\xi \rightarrow \infty$, where the Goldstino equation of motion (32) reduces to that of a Majorana fermion coupled to a scalar field in a curved space-time, whose quantization is also a well known problem of particle production $[8,9]$.

Let us then consider a classical solution to Eq. (32) with helicity $l$, such that in the past $(t \rightarrow-\infty)$, it behaves as a negative-energy plane-wave: i.e.,

$$
\eta_{l}^{p}(x) \rightarrow \frac{1}{a_{i n}^{3 / 2} \sqrt{2 \omega_{i n}^{+}}} e^{i \omega_{i n}^{+} t-i \vec{p} \vec{x}} u(\vec{p}, l),
$$

where $a_{i n}$ is the scale factor at the end of inflation. In the asymptotic future $(t \rightarrow \infty)$, because of the presence of the time-dependent background fields, this solution will no longer behave as a negative-energy mode; rather, it will be a linear superposition of positive and negative frequency modes

$$
\begin{aligned}
\eta_{l}^{p}(x) \rightarrow & \frac{1}{a_{\text {out }}^{3 / 2} \sqrt{2 \omega_{\text {out }}^{+}}}\left(\alpha_{p, l}^{\eta} e^{i \omega_{\text {out }}^{+} t-i \overrightarrow{p x}} u(\vec{p}, l)\right. \\
& \left.+\beta_{-p, l}^{\eta} e^{-i \omega_{\text {out }}^{+} t-i \vec{p} \vec{x}} u^{C}(-\vec{p}, l)\right),
\end{aligned}
$$

where, for a given $\vec{p}$, we have $\left(\omega_{\text {in,out }}^{+}\right)^{2}=\left(m_{\text {in,out }}^{+}\right)^{2}+p^{2}$. The Bogolyubov coefficients satisfy

$$
\left|\alpha_{p, l}^{\eta}\right|^{2}+\left|\beta_{p, l}^{\eta}\right|^{2}=1
$$

Using again our previous result in Eq. (33) we can identify each Fourier mode above with the corresponding Fourier mode for a helicity $\pm 1 / 2$ gravitino (up to a constant). In particular we will find that the Bogolyubov coefficient for the helicity $\pm 1 / 2$ gravitino will be the same as that of the Goldstino, up to $\mathcal{O}(m / p)$, i.e., $\beta_{p, l}^{\psi}=(1+\mathcal{O}(m / p)) \beta_{p, l}^{\eta}$. Notice that because of the different masses of Goldstinos and physical gravitinos, the correction to the Bogolyubov coefficients for gravitinos can depend on time as $\exp \left(i \Delta_{\omega} t\right)$, where $\omega_{\text {out }}^{+}=\omega_{\text {out }}+\Delta_{\omega}$. However, such a term will be relevant only when $t \simeq 1 / \Delta_{\omega}$, that is, much later than the end of preheating. Moreover, remember that the Bogolyubov coefficients are normalized according to Eq. (36) and therefore the proportionality constant is irrelevant.

As a remark, let us note that if we had a renormalizable theory whose low-energy limit is supergravity, we could still use our estimates with the ET, irrespective of the renormalization corrections [19] needed in the complete proof of the theorem. The reason is that we do not need the proportionality constant to obtain the Bogolyubov coefficients.

Therefore the number of gravitinos created with helicity $l= \pm 1 / 2$ and momentum $p, N_{p, l}^{\psi}$ will be given by

$$
N_{p, l}^{\psi}=\left[1+\mathcal{O}\left(\frac{m}{p}\right)\right]\left|\beta_{p, l}^{\eta}\right|^{2}
$$

In conclusion, solving the equation of motion for the Goldstinos in the presence of the external backgrounds, and using Eq. (37), we will obtain the number of helicity $\pm 1 / 2$ gravitinos created during preheating. In the above expression it is explicit that only the knowledge of the solutions in the asymptotic regions is relevant to the particle number calculation.

As an analytic check of the ET, we can compare with the unitary gauge results of [13], obtained in the global supersymmetric limit, $|\phi| \ll M_{P}$. We only have to note that, when $|\phi| \ll M_{P}$, our Goldstino equation in the Landau gauge (32) is reduced to

$$
i \not D \eta-\left(\partial_{\phi} \partial_{\phi} W\right) \eta=0 \text {, }
$$

which is the very same equation obtained in [13] for the helicity $\pm 1 / 2$ gravitinos. Note however, that the condition $|\phi| \ll M_{P}$, is not necessary to prove the ET. Our high-energy result is valid independently of the size of $|\phi|$. 


\section{NUMERICAL EXAMPLE}

As a possible application of the previous results and for further comparison with other works [13], we will study a simple supergravity model based on the superpotential

$$
W=\sqrt{\lambda} \frac{\Phi^{3}}{3} .
$$

Unfortunately, at the minimum of the corresponding inflaton potential, supersymmetry is restored. We will then assume that Eq. (39) is only valid far from the minimum and that close to $\phi=0$ it is modified to satisfy the assumptions needed to apply the ET [see paragraph before Eq. (4)]. For this superpotential, the effective gravitino mass oscillations are not damped in time. Still, we can apply the ET if we take $\phi(t)=0$ for $t \leqslant 0$ and $t \geqslant n T$, where $n$ is an integer (in our example $n=6$ ) and $T$ is the inflaton oscillation period. Despite these problems, we consider this model useful as a numerical illustration of the ET. Hence, we have taken the initial amplitude of the inflaton oscillation to be $\widetilde{\phi}_{0}$ $\simeq 0.2 M_{P}$ (where $\widetilde{\phi}=\sqrt{2} \operatorname{Re} \phi$ is the canonically normalized inflaton), which implies that the effective Goldstino mass is oscillating with amplitude $m_{G} \simeq \sqrt{2 \lambda} M$. With these initial and final conditions we have calculated numerically the number of Goldstinos produced from Eq. (32), using the standard results for the production of fermions obtained in [8]. We thus look for solutions of the Goldstino equation with momentum $p$ and helicity $l$ of the form

$$
\eta^{p l}(x)=a^{-3 / 2}(t) e^{i \vec{p} \cdot \vec{x}} U^{\overrightarrow{p l}}(t),
$$

with

$$
\begin{aligned}
U^{\overrightarrow{p l}}(t)= & \frac{1}{\sqrt{\omega_{\text {in }}+m_{i n}}}\left[i \gamma^{0} \partial_{0}-\vec{p} \cdot \vec{\gamma}+a(t)\left(e ^ { G / 2 } \left(G_{, \phi \phi}\right.\right.\right. \\
& \left.\left.\left.+G_{, \phi}^{2}\right)\right)\right] f_{p l}(t) u(\vec{p}, s) .
\end{aligned}
$$

Using the above ansatz, we can write the equation as follows:

$$
\begin{gathered}
{\left[\frac{d^{2}}{d \widetilde{t}^{2}}+\kappa^{2}+\frac{i}{\sqrt{\lambda}} \frac{d}{d \tilde{t}}\left(b e^{G / 2}\left(G_{, \phi \phi}+G_{, \phi}^{2}\right)\right)\right.} \\
\left.+\frac{b^{2}}{\lambda} e^{G}\left(G_{, \phi \phi}+G_{, \phi}^{2}\right)^{2}\right] f_{\kappa l}(\tilde{t})=0
\end{gathered}
$$

with $\kappa=p /\left(a_{i n} \sqrt{\lambda}\right)$ and $\tilde{t}=a_{i n} \sqrt{\lambda} t$ and the new scale factor is defined as $b(\tilde{t})=a(\tilde{t}) / a_{i n}$. The initial conditions are $f_{\kappa l}(0)=1$ and $\dot{f}_{\kappa l}(0)=-i \kappa$. In particular, for the Goldstino occupation number we find

$$
N_{\kappa l}^{\eta}(n T)=\frac{1}{4 \kappa}\left(2 \kappa+i\left[\dot{f}_{\kappa l}^{*}(n T) f_{\kappa l}(n T)-f_{\kappa l}^{*}(n T) \dot{f}_{\kappa l}(n T)\right]\right) .
$$
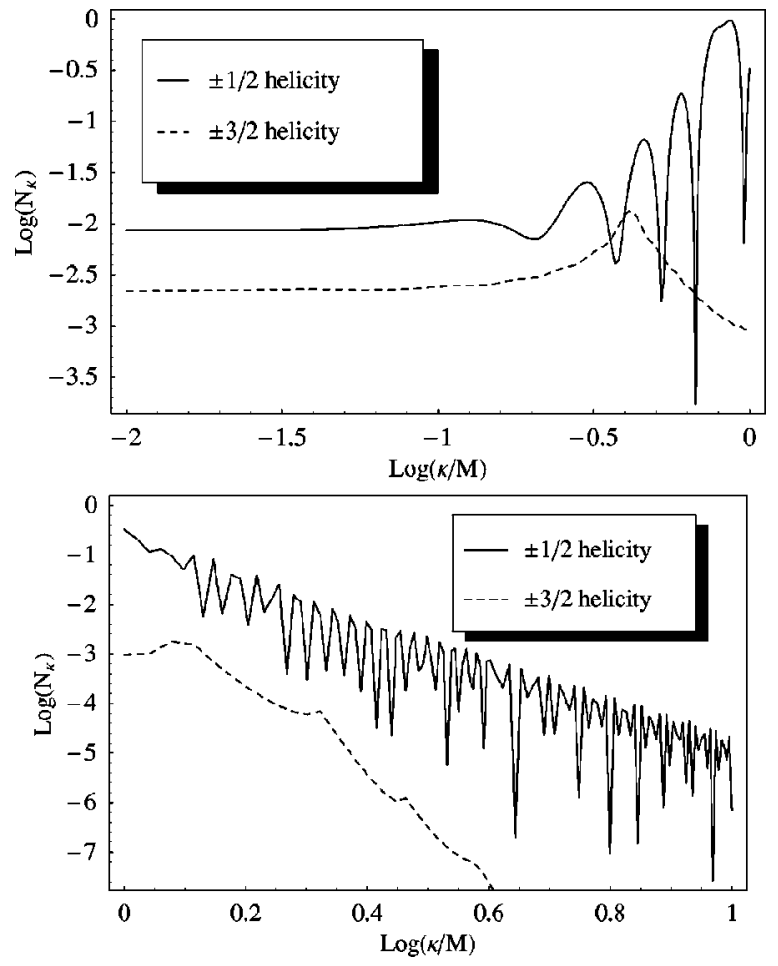

FIG. 1. Spectrum of helicity $\pm 3 / 2$ and helicity $\pm 1 / 2$ gravitinos for different ranges of momentum. The helicity $\pm 1 / 2$ production has been obtained using the ET.

Using Eq. (37), we get the occupation number of helicity $\pm 1 / 2$ gravitinos directly from Eq. (43), whereas for those with helicity $\pm 3 / 2$ we use Eq. (6) following the same steps as before [10]. In Fig. 1, we have plotted both spectra and we see that for this particular model, the production of helicity $\pm 3 / 2$ gravitinos is suppressed by two to three orders of magnitude with respect to the $\pm 1 / 2$ gravitinos. Note that $N_{k l}$ depends on the number of oscillations and on the inflaton initial conditions. Thus, we cannot make a comparison for the whole spectrum with the unitary gauge estimations obtained in the first references in [13] and the complete numerical analysis of the last reference in [13] (made without the ET, although they have checked that the formulas hold in one particular case). Nevertheless, the ET tells us that, since the mass of the Goldstino will be less than $a(t) \sqrt{\lambda}$, both results should agree for $p \gg a(n T) \sqrt{\lambda}$, i.e., with the above definitions $\kappa \gg b(n T)$ [in our example $b(n T) \simeq 12$ ], irrespectively of the initial conditions. Indeed, the orders of magnitude are in good agreement with the previous works.

However, there are some other models in which the application of the ET is somewhat restricted, such as a pure quadratic superpotential $W=m_{\phi} \Phi^{2}$. In this case, at the minimum of the potential, supersymmetry is restored and therefore the definition of helicity $\pm 1 / 2$ gravitinos is meaningless. Ignoring this problem, although the amplitude of the inflaton oscillations is damped, the Goldstino mass term, which contains the second derivative of the superpotential, tends to a constant of $\mathcal{O}\left(m_{\phi}\right)$, typically much larger than $m_{3 / 2}$. Then, the ET will be useful to calculate only that portion of the spectrum with energy much higher than the inflaton mass. 
Finally, there is an additional condition to obtain reliable predictions. Since gravitinos are produced in large numbers, with vastly different energies, we have to estimate what fraction of them does not satisfy the ET conditions. For that purpose we define the number density of gravitinos produced with both helicities as

$$
n(t)=\frac{1}{\pi^{2} a^{3}(t)} \int_{0}^{\infty} N_{p, l} p^{2} d p .
$$

Then, for those gravitinos with momenta lower than their mass, i.e., $p^{2}<a_{\text {out }}^{2} m_{3 / 2}^{2}$, the ET does not apply. Thus the number density of "excluded" gravitinos is

$$
n(t)_{\text {exc }} \leqslant \frac{1}{3 \pi^{2} a^{3}(t)} a_{\text {out }}^{3} m_{3 / 2}^{3} .
$$

In addition, we can estimate the total number density of produced gravitinos as

$$
n(t)_{t o t} \simeq \frac{1}{3 \pi^{2} a^{3}(t)} a_{i n}^{3} m_{\phi}^{3} .
$$

Hence we obtain that the fraction of gravitinos that do not satisfy the ET applicability conditions is

$$
\frac{n_{\text {exc }}}{n_{\text {tot }}} \leqslant\left(\frac{a_{\text {out }} m_{3 / 2}}{a_{\text {in }} m_{\phi}}\right)^{3} .
$$

Accordingly, the additional ET applicability condition is: $a_{\text {out }} m_{3 / 2} \ll a_{i n} m_{\phi}$. We see that the result depends on the duration of the preheating era and the ratio of gravitino and inflaton masses. Typically, the production takes place in a few inflaton oscillations, which implies that the scale factor only grows by a few orders of magnitude, not enough to overcome the mass difference. Therefore, in these models, the ET safely describes the production of the vast majority of helicity $\pm 1 / 2$ gravitinos.

\section{CONCLUSIONS}

We have studied the production of helicity $\pm 1 / 2$ gravitinos using the equivalence of Goldstinos and gravitinos at high energies. We have shown that in the $R_{\xi}$ gauges, where the Goldstino and gravitino equations of motion decouple, the classical solutions of the Goldstino equation are proportional to those of the helicity $\pm 1 / 2$ gravitinos in the asymptotic static regions. This result is sufficient to relate the production of Goldstinos to the production of helicity $\pm 1 / 2$ gravitinos. Furthermore, we have shown how in the Landau gauge, the equation of motion for the Goldstino is considerably simpler. As a check, we have compared our results with previous ones obtained in the unitary gauge and we have found good agreement in the equivalence theorem applicability regions.

We have clearly identified the ET applicability conditions in the context of gravitino production: (i) The frequency of the inflaton field oscillations should be larger than the gravitino mass, $m_{\phi} \gg m_{3 / 2}$. If we are interested in the pure gravitational production, then one should also require $H \gg m_{3 / 2}$. These conditions ensure that the typical energy of the produced particles will be larger than their masses. (ii) The sources should vanish asymptotically, which implies that the space-time curvature should decrease with time and also that the amplitude of the inflaton oscillations should be damped. (iii) The ET calculations are only useful if most of the gravitinos have a large enough energy, which requires $a_{\text {out }} m_{3 / 2}$ $\ll a_{i n} m_{\phi}$.

Concerning the potential applications of our results, we have shown that cubic superpotentials, with a slight modification in their form, together with appropriate initial conditions, could satisfy the above applicability requirements. More elaborated models would require to extend the present proof to more than one chiral multiplet and non-minimal supergravity. Further work along this line is in progress.

\section{ACKNOWLEDGMENTS}

A.L.M. wishes to acknowledge very useful discussions with A. Linde, R. Kallosh and A. Mazumdar. J.R.P. thanks the CERN Theory Division, where part of this work was carried out, for their hospitality. This work has been partially supported by the Spanish Ministerio de Educación y Ciencia under project CICYT AEN97-1693. A.L.M. also acknowledges the Spanish Ministerio de Educación y Ciencia for financial support.
[1] H. P. Nilles, Phys. Rep. 110, 1 (1984).

[2] D. Bailin and A. Love, Supersymmetric Gauge Field Theory and String Theory (IOP, Bristol, 1994).

[3] E. W. Kolb and M. S. Turner, The Early Universe (AddisonWesley, New York, 1990).

[4] J. Ellis, A. Linde, and D. Nanopoulos, Phys. Lett. 118B, 59 (1982).

[5] S. Sarkar, Rep. Prog. Phys. 59, 1493 (1996).

[6] J. Ellis, J. E. Kim, and D. V. Nanopoulos, Phys. Lett. 145B, 181 (1984).

[7] L. Kofman, A. D. Linde, and A. A. Starobinsky, Phys. Rev. Lett. 73, 3195 (1994); Phys. Rev. D 56, 3258 (1997); J. H.
Traschen and R. H. Brandenberger, ibid. 42, 2491 (1990); Y. Shtanov, J. Traschen, and R. Brandenberger, ibid. 51, 5438 (1995).

[8] J. Baacke, K. Heitmann, and C. Patzold, Phys. Rev. D 58, 125013 (1998).

[9] P. B. Greene and L. Kofman, Phys. Lett. B 448, 6 (1999).

[10] A. L. Maroto and A. Mazumdar, Phys. Rev. Lett. 84, 1655 (2000).

[11] A. L. Maroto, Nucl. Phys. B (Proc. Suppl.) 81, 351 (2000).

[12] M. Lemoine, Phys. Rev. D 60, 103522 (1999).

[13] R. Kallosh, L. Kofman, A. Linde, and A. Van Proeyen, Phys. Rev. D (to be published), hep-th/9907124; G. F. Giudice; A. 
Riotto and I. Tkachev, J. High Energy Phys. 08, 009 (1999); G. F. Giudice, A. Riotto, and I. Tkachev, ibid. 11, 036 (1999).

[14] D. H. Lyth, D. Roberts, and M. Smith, Phys. Rev. D 57, 7120 (1998); D. H. Lyth, hep-ph/9909387; hep-ph/9911257.

[15] R. Casalbuoni, S. De Curtis, D. Dominici, F. Feruglio, and R. Gatto, Phys. Lett. B 215, 313 (1988); Phys. Rev. D 39, 2281 (1989).

[16] J. M. Cornwall, D. N. Levin, and G. Tiktopoulos, Phys. Rev. D 10, 1145 (1974).

[17] C. E. Vayonakis, Lett. Nuovo Cimento Soc. Ital. Fis. 19, 383 (1976); B. W. Lee, C. Quigg, and H. Thacker, Phys. Rev. D 16, 1519 (1977).

[18] M. S. Chanowitz and M. K. Gaillard, Nucl. Phys. B261, 379 (1985).

[19] Y. P. Yao and C. P. Yuan, Phys. Rev. D 38, 2237 (1988); J. Bagger and C. Schmidt, ibid. 41, 264 (1990); H. J. He, Y. P. Kuang, and X. Li, Phys. Rev. Lett. 69, 2619 (1992).

[20] H. J. He, Y. P. Kuang, and X. Li, Phys. Rev. D 49, 4842
(1994).

[21] E. Cremmer, B. Julia, J. Scherk, S. Ferrara, L. Girardello, and P. van Nieuwenhuizen, Nucl. Phys. B147, 105 (1979).

[22] P. Holman, P. Ramond, and G. G. Ross, Phys. Lett. 137B, 343 (1984).

[23] G. G. Ross and S. Sarkar, Nucl. Phys. B461, 597 (1996).

[24] L. Baulieu, A. Georges, and S. Ouvry, Nucl. Phys. B273, 366 (1986).

[25] N. D. Birrell and P. C. W. Davies, Quantum Fields in Curved Space (Cambridge University Press, England, 1982).

[26] T. Moroi, Ph.D. thesis, Tohoku University, 1995, hep-ph/9503210.

[27] V. M. Mostepanenko and V. M. Frolov, Yad. Fiz. 19, 885 (1974) [Sov. J. Nucl. Phys. 19, 451 (1974)]; A. A. Grib, S. G. Mamayev, and V. M. Mostepanenko, Vacuum Quantum Effects in Strong Fields (Friedmann Laboratory Publishing, St. Petersburg, 1994).

[28] P. Fayet, Phys. Lett. 70B, 461 (1977). 